\title{
Motivação para mudança: análise fatorial da URICA para hábitos alimentares
}

\author{
SimmeAmmtanoBittencart- Pontifía UnivesidadeCatálica doRioGrandedbSul, PatoAlege, RioGrandedbSul, Brasil \\ Pada Lucena dosSantos- Pontifía UnivesidadeCatdica doRioGrandedbSul, PatoAlege RioGrandedbSul, Brasil \\ Margarthda Silva Olivera - Pontifía UnivesidadeCatdica dbRioGrandedbSul, PatoAlege, RioGrandedbSul, Brasil
}

\begin{abstract}
Resumo
O presente estudo objetivou realizar a adaptação da URICA para avaliação da motivação para a mudança de hábitos alimentares e posterior redução da versão de 32 para a de 24 itens, mediante análise fatorial exploratória e de índices de fidedignidade. Trata-se de um estudo de cunho instrumental, realizado com 175 participantes de programas de redução de peso do sul do Brasil. A análise fatorial apontou uma solução de 4 fatores, referentes aos 4 estágios de motivação para a mudança e apresentou um alpha de Cronbach total de 0,842, que variou de 0,813 a 0,940 entre os 4 domínios.

Palavasdhave Analise fatorial; Comportamento alimentar; URICA; Mudança; Motivação.
\end{abstract}

Motivation for change: factor analysis of the URICA for eating habits

\begin{abstract}
This study aimed to perform the adaptation of URICA to assess motivation to change eating habits and the subsequent reduction from 32 to 24 items through exploratory factor analysis and reliability indices. This is an instrumental nature study, conducted with 175 participants in weight reduction programs from the South of Brazil. The factor analysis yielded a solution of four factors, relating to the four stages of motivation for change and Cronbach's Alpha showed a total of 0.84 , which ranged from 0.814 to 0.94 among the four domains.
\end{abstract}

Keguads Factor analysis; Feeding practices; URICA; Change; Motivation.

\section{Motivación para cambio: análisis factorial de la URICA para hábitos alimentarios}

\begin{abstract}
Resumen
El presente estudio objetivó realizar la adaptación de la URICA para evaluación de la motivación para el cambio de hábitos alimentarios y posterior reducción de la versión de 32 para 24 ítems, a través de análisis factorial exploratorio y de índices de fiabilidad. Se trata de un estudio de carácter instrumental, realizado con 175 participantes de programas de reducción de peso del sur de Brasil. El análisis factorial apuntó una solución de 4 factores, referentes a los 4 estadios de motivación para el cambio y presentó un Alpha de Cronbach total de 0,842, que varió de 0,813 a 0,940 entre los 4 dominios.

Palabras dave Análisis factorial; Comportamiento alimentar; URICA; Cambio; Motivación.
\end{abstract}

A obesidade é uma entidade nosológica distinta que, assim como os transtornos alimentares, está associada aos hábitos de alimentação de seus portadores. O número de obesos aumentou significativamente, o que elevou a obesidade ao status de epidemia mundial e de grave problema de saúde pública (Finucane \& cols., 2011). Tanto a anorexia nervosa (NA) como a bulimia nervosa (BN) são transtornos alimentares crônicos e estão associados a um alto índice de comorbidades. Uma vez que os transtornos alimentares têm uma etiologia multifatorial, necessita-se de avaliações e abordagens que contemplem a complexidade dos fatores envolvidos tanto em sua gênese quanto em sua manutenção (Steinhausen, 2009).

É importante salientar que os modelos de causa única etiológica têm se mostrado inadequados para explicar a adoção e a mudança de comportamentos, necessitando-se de modelos complexos que expliquem tanto a diversidade como aspectos em comum, apresentados (Diclemente, 2003). O Modelo Transteórico de Mudança de Comportamento (MTT) foi elaborado com a finalidade de abarcar a complexidade do processo de motivação para mudança de comportamento e da busca de hábitos saudáveis (Diclemente, 2003). 0 MTT é um modelo passível de ser aplicado em uma grande variedade de comportamentos, sendo utilizado atualmente em diversas áreas (Levesque, Gelles \& Velicer, 2000), como modificação de hábitos alimentares, estratégias para controle do diabetes e do peso, comportamentos ligados ao uso de substâncias psicoativas, prática de atividades físicas, manifestação de distúrbios de ansiedade, prevenção de diferentes tipos de câncer, gravidez não-planejada, HIV/AIDS, dentre outras (Rossi \& cols., 2001).

Um dos pressupostos principais do MTT é que as pessoas passam por diferentes estágios de motivação para mudança, durante a modificação de um comportamento problema. Pela identificação do estágio de mudança no qual a pessoa se encontra em determinado momento, é possível saber 0 quanto 0 sujeito está motivado para efetivamente mudar, sendo essa avaliação decisiva no processo de escolha de estratégias terapêuticas mais adequadas e eficazes para 0 aumento e manutenção da motivação, favorecendo a mudança efetiva de comportamento. Segue a descrição 
dos estagios motivacionais, segundo DiClemente (2003):

(1) Pré-contemplação: nesse estágio a pessoa sequer consegue identificar que tem um problema e não mostra intenção de mudar o seu comportamento. (2) Contemplação: aqui 0 indivíduo já consegue reconhecer que tem um problema e apresenta um alto nível de ambivalência. Nesse momento as pessoas se mostram preocupadas e avaliam as vantagens e desvantagens de mudar. (3) Ação: nesse estágio a pessoa já apresenta ações específicas na direção da mudança de comportamento. (4) Manutenção: esse último estágio caracteriza-se pela manutenção da mudança, onde se consolidam todos os ganhos obtidos nos estágios anteriores.

Os estágios motivacionais podem ser identificados por meio de alguns instrumentos, como: a University of Rhode Isand Change Assesment (McConnughy, Prochaska \& Velicer, 1983), representada pela sigla URICA, a Stages of Change Readiness and Treatment Eagemess Scale (Figlie, Dunn, Bazan \& Laranjeira, 2005), abreviada pelo termo SOCRATES, além de algoritmos e escalas analógicovisuais, como a Régua de Prontidão (Velásquez, Maurer, Crouch \& DiClemente, 2001).

A URICA é um instrumento que tem gerado algum suporte para a validade preditiva dos estágios de mudança em tratamentos na área da saúde, uma vez que já previu eficazmente resultados de diversos programas (D ozois, Westra, Collins, Fung \& Garry, 2004). O s itens dessa escala são divididos em quatro subescalas, correspondentes aos estágios de PréContemplação, Contemplação, Ação e Manutenção. 0 instrumento é autoaplicável sendo que, para cada item, existem 5 alternativas de respostas em escala Liket, de discordo totalmente a concordo totalmente. Algumas versões da URICA, atualmente existentes, destinam-se a avaliar a motivação para a mudança de comportamentos específicos, como é o dos estudos de validação da URICA para usuários de cocaína e álcool (Pantalon, Nich, Frankforter \& Carroll, 2002), alcoolistas internados (Willoughby \& Edens, 1996), pessoas com problemas relacionados à ansiedade (Dozois \& cols., 2004), tabagistas (Amodei \& Lamb, 2004; Velicer, Diclemente, Prochaska \& Brandenburg, 1985), pessoas com transtornos alimentares (Hasler, Klaghofer \& Buddeberg, 2003), comedores compulsivos (Dunn, Neighbors \& Larimer, 2003), realização de exercícios físicos (Lerdal \& cols., 2009), entre outros comportamentos. Evidências de validade da URICA já foram estudadas em diversos países, tais como Alemanha (Hasler \& cols., 2003), Canadá (Dozois \& cols., 2004), Estados Unidos (Amodei \&
Lamb, 2004) e Noruega (Lerdal \& cols., 2009), dada a repercussão e relevância dessa escala.

A versão original da URICA foi validada para o comportamento específico de uso de álcool e possuía 32 itens (Carney \& Kivlaham, 1995). Atualmente existem versões do instrumento que variam de 34 a 12 itens (Belding, Iguchi \& Lamb, 1996; Carbonari \& DiClemente, 2000; Carbonari, DiClemente \& Zweben, 1994; Carney \& Kivlaham, 1995; Pantalon \& cols., 2002; Siegal, Li, Rapp \& Saha, 2001). No Brasil, a URICA 24 itens já foi adaptada e teve suas evidências de validade estudadas para o uso de álcool, tabaco, drogas ilícitas e jogo patológico, com alfas de Cronbach totais variando de 0,65 a 0,82 (Oliveira, Freire \& Cazassa, 2007; Rodrigues, Oliveira, Ludwig, Cazassa \& Fernandes, 2007; Souza, Silva \& Oliveira, no prelo; Szupszynski \& O liveira, 2008). No entanto, até o momento, não existe no Brasil nenhuma versão da URICA para a mudança de hábitos alimentares.

Dada a importância da URICA como instrumento capaz de auxiliar no monitoramento terapêutico e de avaliar a motivação de pacientes para mudar seus hábitos alimentares, este estudo se propõe a adaptar 0 instrumento para a avaliação da motivação para a mudança de hábitos alimentares. Além disso, busca-se a redução do número de itens da escala, de 32 para 24 itens. Por meio desse processo, serão também avaliadas evidências de validade da estrutura interna da nova versão reduzida.

\section{Deineamento}

\section{Método}

Trata-se de um estudo com delineamento transversal, tendo em vista que as variáveis foram medidas em um único espaço temporal, e de cunho instrumental (Carretero-Dios \& Pérez, 2007). Estudos instrumentais envolvem tanto a adaptação de testes, como 0 estudo de suas propriedades psicométricas (Montero \& León, 2005).

\section{Instrumentos}

Para a efetivação do presente estudo, cada participante respondeu aos instrumentos abaixo apresentados:

Entrevista estruturada: foi elaborada exclusivamente para este estudo, com o objetivo de obter dados para caracterização amostral. Nessa entrevista foram incluídos os Critérios de Classificação Econômica Brasil (ANEP, 2008), para classificação de cada participante em diferentes classes econômicas, na ordem de maior a menor poder aquisitivo (variando da classe A para a classe E, respectivamente). Semelhantemente, na entrevista estruturada foram colhidas informações a respeito do IMC (Índice de 
Massa Corporal) de cada participante. Para tanto, consideraram-se os critérios classificatórios adotados pela Organização Mundial de Saúde (WHO , 2006), que prevê que este seja calculado pelo peso em quilogramas dividido pelo quadrado da altura em metros $\left(\mathrm{kg} / \mathrm{m}^{2}\right)$.

URICA (McConnaughy \& cols., 1983): a URICA possui diferentes versões, de acordo com o número de itens. Neste estudo, a versão de 32 itens foi adaptada, para avaliar a motivação para a mudança de hábitos alimentares e, posteriormente, aplicada nos participantes, objetivando sua redução posterior para a versão de 24 itens, medidante de estudo de suas propriedades psicométricas.

\section{Análisedos dados}

Os dados foram computados no banco de dados SPSS versão 17.0. Para análise dos dados utilizou-se estatística descritiva e inferencial (alfa de Cronbach; análise dos componentes principais, com rotação varimax e normalização de Kaiser; Teste de KaiserMeyer-Olkin; Teste de Esfericidade de Barlett, todos com nível de significância de 5\%).

A URICA 32 itens é composta de 4 subescalas (relativas aos 4 estágios motivacionais), as quais são compostas por 8 itens distintos. Nesse sentido, as hipóteses deste estudo, de acordo com o que é previsto teoricamente, são de que, pela análise fatorial exploratória dos dados, obtenham-se 4 fatores, com no mínimo 6 itens cada, os quais devem avaliar o estágio motivacional que cada domínio abrange. Assim, para critérios de retenção dos fatores, utilizou-se 0 critério de Kaiser (autovalor igual ou maior a 1,0), o critério de Catell (scee pld) e, ainda, cada fator, para ser retido, deve ser constituído de no mínimo 6 itens (conforme é esperado teoricamente) com cargas fatoriais e comunalidades iguais ou maiores que 0,30 (Hair, Black, Babin, Anderson \& Tatham, 2009).

\section{Aspetos éticos eprocedimatos}

O presente projeto foi submetido e aprovado pelo Comitê de Ética em Pesquisa da PUCRS (sob ofício nํㅡ 364/ 05-CEP). Todos os sujeitos convidados a participar deste estudo eram participantes de programas ambulatoriais de redução de peso. Foram convidados a participar do estudo de forma individual, por integrantes da equipe de pesquisa, ocasião na qual foram devidamente informados a respeito da natureza e dos propósitos do estudo, além da responsabilidade da equipe de investigação quanto ao sigilo dos dados coletados. Todos os sujeitos de pesquisa assinaram 0 Termo de Consentimento Livre e Esclarecido. As aplicações do protocolo de pesquisa foram feitas de forma individual, por pesquisadores devidamente treinados.
Amostra

A amostra foi composta por 175 participantes. Do total, 93,7\% (164) eram mulheres. A média de idade foi de 41,05 anos (DP=13,10). D os participantes, $36,6 \%$ (64) estavam na faixa do ensino médio, 52\% (91) se encontravam na faixa do ensino superior e apenas $11,4 \%$ (20) na faixa do ensino fundamental. Quanto ao estado civil, 54\% (95) dos participantes eram casados, 34\% (60) solteiros, 10,4\% (18) separados e $1,6 \%$ (2) viúvos. De acordo com os Critérios de Classificação Econômica Brasil (ANEP, 2008), a maior parte dos participantes, 40,6\% (71), encontrava-se na classe $C$, seguidos de 38,8\% (68) que estavam na classe B, 10,9\% (19) na classe A e 9,7\% (17) nas classes D ou E. Segundo o cálculo do IMC (Índice de Massa Corporal), 16\% (28) dos participantes apresentavam IMC normal, 32,6\% (57) sobrepeso, 29,1\% (51) apresentavam obesidade grau 1 (risco moderado de comorbidades), 10,9\% (19) obesidade grau 2 (risco grave de comorbidades) e 11,4\% (20) obesidade grau 3 (risco muito grave de comorbidades). Assim, do total da amostra, 51,4\% (90) apresentavam algum grau de obesidade, segundo pontos de corte da Organização Mundial de Saúde (WHO , 2000).

Os critérios de inclusão dos participantes foram os seguintes: participar de um programa de tratamento, com 0 foco na modificação de hábitos alimentares, das cidades da Grande Porto Alegre; ter entre 18 e 60 anos e ter concluído, no mínimo, 5 anos de estudo formal. 0 tamanho amostral final foi calculado em função do número de itens da escala (24 itens), sendo necessários pelo menos 5 sujeitos para cada variável, 0 que equivaleria a um número mínimo de 120 participantes. Além disso, para a realização da análise fatorial, 0 recomendado é que o tamanho da amostra seja igual ou maior que 100 participantes (Hair \& cols., 2009).

\section{Resultados}

\section{Adaptaçãodbinstrumento}

No que se refere ao processo de adaptação transcultural de um instrumento, este deve levar em conta cada comportamento específico, adaptando a linguagem de cada item do instrumento para 0 comportamento a ser avaliado, o que garante maior adequação das escalas (Nunes \& Primi, 2010), proporcionando o estudo de evidências de validade de conteúdo ao longo desse processo. A URICA 32 itens foi traduzida da língua inglesa (original) para a língua portuguesa. Os procedimentos, nessa primeira fase, ocorreram na seguinte ordem: tradução e badktrandation (com a devida adaptação da linguagem de modo que pudesse ser usada para avaliar a motivação para a mudança de hábitos alimentares), avaliação de 
comitê de inter juízes e aplicação piloto. Após, a URICA 32 itens foi aplicada em uma amostra piloto de 20 participantes para a avaliação da compreensão gramatical. Houve adequada compreensão das sentenças pelos sujeitos, uma vez que nenhum dos 20 participantes mostrou dificuldades nesse quesito. Posteriormente, foram excluídos os protocolos de pesquisa dessa primeira fase, sendo, então, iniciada a coleta de dados efetiva deste estudo.

\section{Análisefatoial explaratóia}

Durante a realização de estudos que visem observar as evidências de validade de uma escala, é essencial efetuar a análise de sua estrutura interna. Uma das formas de se avaliar esse tipo de evidência é pela a análise fatorial, uma técnica estatística utilizada para identificar quantos fatores determinado instrumento está medindo e quais itens representam cada fator (Urbina, 2007). Esse tipo de análise produz importantes resultados, por meio dos quais é possível tomar decisões a respeito da qualidade e quantidade dos itens que compõem o instrumento, assim como a respeito do instrumento como um todo (Pasquali, 2010).

Para a realização da análise fatorial de um conjunto existente de variáveis, deve-se primeiramente proceder à análise da adequação dos dados, para que seja realizado esse tratamento estatístico. A matriz de dados deve ter correlações suficientes para que se justifique a realização da análise fatorial. $\mathrm{Na}$ análise visual da matriz de correlações, deve haver um número considerável de correlações maiores que 0,30. Uma maneira de examinar a matriz de correlação inteira é por meio do Teste Barlett de Esfericidade, que determina a probabilidade de que a matriz tenha correlações significativas entre as variáveis. Além do Teste de Barlett, há o teste de adequação da amostra de
Kaiser-Meyer-Olkin (KMO), que quantifica o grau de intercorrelações entre as variáveis e a adequação da análise fatorial. $0 \mathrm{KMO}$ é um índice que varia de 0 a 1 , sendo considerado inaceitável, abaixo de 0,50. Neste estudo, o índice KMO foi de 0,88 e os achados obtidos por meio do Teste de Esfericidade de Barlett, foram significativos ( $\left.\mathrm{p}<0,001 ; \chi^{2}=3410,40 ; \mathrm{d}=496\right), 0$ que indica que os dados são adequados para a realização da análise fatorial.

A análise fatorial exploratória dos 32 itens foi realizada mediante análise dos componentes principais, com rotações varimax e normalização de Kaiser, objetivando a replicação do tipo de tratamento estatístico realizado no estudo original do instrumento (McConnaughy \& cols., 1983). A solução inicial extraiu 6 fatores com autovalores iguais ou maiores que 1 , porém o quinto e o sexto não atenderam aos critérios de retenção pré estabelecidos. Mediante a análise do gráfico scre verificou-se que uma solução de 4 fatores seria a que abrangeria maior percentual da variância total, com maior parcimonialidade. Dada esta configuração, realizou-se nova análise dos 32 itens, com extração de 4 fatores, correspondente ao número de domínios que atendeu aos critérios de retenção. Os autovalores encontrados para cada um dos 4 fatores foram 9,63; 4,24; 3,10 e 1,43, os quais explicavam $30,12 \% ; \quad 13,27 \%, 9,69 \%$ e $4,417 \%$ da variância, perfazendo um total de $57,55 \%$ da variância total.

Após análise da matriz rotacionada e interpretação dos fatores, os mesmos foram assim nomeados, de acordo com os nomes dos estágios motivacionais: Fator 1: Ação; Fator 2: Contemplação; Fator 3: PréContemplação; e Fator 4: Manutenção. Na Tabela 1, os 4 fatores, com respectivas comunalidades e cargas dos itens rotacionados podem ser observados.

Tabela 1. Análise fatorial exploratória para redução da URICA de 32 para 24 Itens

(continua)

\begin{tabular}{ccccccc}
\hline \multicolumn{2}{c}{ URICA versão 32 itens } & Fator 1 & Fator 2 & Fator 3 & Fator 4 \\
\hline $\begin{array}{c}\text { Item de cada estágio } \\
\text { motivacional }\end{array}$ & $\begin{array}{c}\text { Comunalidades } \\
\text { dos itens }\end{array}$ & Ação & Contemplação & $\begin{array}{c}\text { Pré- } \\
\text { Contemplação }\end{array}$ & Manutenção \\
\hline Ação & 25 & 0,802 & 0,889 & & & \\
& 17 & 0,788 & 0,873 & & \\
30 & 0,736 & 0,857 & & \\
14 & 0,734 & 0,853 & & \\
20 & 0,746 & 0,830 & & \\
7 & 0,751 & 0,816 & & \\
$3^{*}$ & 0,536 & $0,642^{* *}$ & $0,304^{* *}$ & & \\
$10^{*}$ & 0,672 & & $0,742 \mathrm{I}$ & & \\
\hline
\end{tabular}


Tabela 1. Análise fatorial exploratória para redução da URICA de 32 para 24 Itens

\begin{tabular}{|c|c|c|c|c|c|c|}
\hline \multicolumn{3}{|c|}{ URICA versão 32 itens } & \multirow{2}{*}{$\begin{array}{l}\text { Fator } 1 \\
\text { Ação }\end{array}$} & \multirow{2}{*}{$\begin{array}{c}\text { Fator } 2 \\
\text { Contemplação }\end{array}$} & \multirow{2}{*}{$\begin{array}{c}\text { Fator 3 } \\
\text { Pré- } \\
\text { Contemplação }\end{array}$} & \multirow{2}{*}{$\begin{array}{c}\text { Fator } 4 \\
\text { Manutençãc }\end{array}$} \\
\hline $\begin{array}{r}\text { Item de cad } \\
\text { motivac }\end{array}$ & & $\begin{array}{c}\text { Comunalidades } \\
\text { dos itens }\end{array}$ & & & & \\
\hline \multirow[t]{8}{*}{ Contemplação } & 15 & 0,665 & & 0,779 & & \\
\hline & 12 & 0,673 & & 0,760 & & \\
\hline & 8 & 0,596 & & 0,732 & & \\
\hline & 4 & 0,659 & $0,333^{* *}$ & $0,669 * *$ & $-0,300 * *$ & \\
\hline & 19 & 0,595 & & $0,601 * *$ & & $0,482^{* *}$ \\
\hline & 21 & 0,42 & & $0,508 * *$ & & $0,309 * *$ \\
\hline & $24^{*}$ & 0,491 & & & $0,592 * *$ & $0,341^{* *} \mathrm{I}$ \\
\hline & $2^{*}$ & 0,413 & $0,599 \mathrm{t}$ & & & \\
\hline \multirow{8}{*}{$\begin{array}{c}\text { Pré- } \\
\text { Contemplação }\end{array}$} & 29 & 0,648 & & & 0,773 & \\
\hline & 31 & 0,655 & & & 0,756 & \\
\hline & 13 & 0,588 & & & 0,722 & \\
\hline & 23 & 0,481 & & & 0,693 & \\
\hline & 5 & 0,576 & & $-0,461 * *$ & $0,595 * *$ & \\
\hline & 1 & 0,471 & & $-0,394 * *$ & $0,544^{* *}$ & \\
\hline & $26^{*}$ & 0,403 & & & & $0,614 \mathrm{I}$ \\
\hline & $11 *$ & 0,054 & H & H & If & If \\
\hline \multirow[t]{8}{*}{ Manutenção } & 27 & 0,565 & & $0,370 * *$ & & $0,433^{* *}$ \\
\hline & 16 & 0,626 & $0,337 * *$ & & & $0,348 * *$ \\
\hline & 32 & 0,422 & & $0,524^{* *}$ & & $0,333^{* *}$ \\
\hline & 28 & 0,589 & & $0,307 * *$ & & $0,673 * *$ \\
\hline & 18 & 0,643 & $0,476^{* *}$ & $0,383^{* *}$ & & $0,633^{* *}$ \\
\hline & 22 & 0,671 & & $0,396 * *$ & & $0,531^{* *}$ \\
\hline & $6^{*}$ & 0,449 & $\mathrm{H}$ & $\mathrm{H}$ & $\mathrm{H}$ & $\mathrm{H}$ \\
\hline & 9* & 0,294 & $\mathrm{H}$ & $\mathrm{H}$ & & $\mathrm{Ht}$ \\
\hline
\end{tabular}

Análise dos componentes principais, com rotação varimax e normalização de Kaiser

* Itens que serão excluídos

** Carga maior que 0,30 em mais de 1 fator

千 Item não carregou no fator que se propõe a avaliar

¥¥ Não carregou em nenhum dos fatores

\section{Reeurãodositens}

Com base na análise dos fatores e respectivos itens, comunalidades e cargas fatoriais, se decidiu, quanto a cada fator: 1 (Ação): excluir o item 10, visto que não carregou no fator que se propõe a avaliar e 0 item 3 visto que, dentre os demais itens deste fator, é 0 que apresenta carga mais baixa. Além disso, o item três carregou em mais de um fator, enquanto os demais itens carregaram somente no fator que se propõem a avaliar. 02 (Contemplação): excluir os itens 2 e 24, visto que nenhum dos dois apresentou carga igual ou maior a 0,30 no fator que se propõem a avaliar. 03 (Pré-Contemplação): excluir os itens 26 e 11, visto que nenhum dos dois carregou no domínio que se destina a mensurar. Além disso, a comunalidade do item 11 é menor que 0,30. E, o 4 (Manutenção): Excluir os itens 6 e 9 , uma vez que nenhum deles carregou no fator.

\section{Análiseda consistênaia intema}

A estrutura interna de um instrumento pode ser avaliada pelo cálculo de confiabilidade, a qual é também denominada de precisão e de fidedignidade e se refere à estabilidade do teste, de maneira que, se uma mesma pessoa for avaliada por dois diferentes aplicadores, os resultados deverão ser bem próximos, visto que 0 teste é o mesmo e continua avaliando a mesma coisa. Quanto mais preciso for considerado determinado teste, menos erros de medida ele contém. 
De maneira geral, se considera que para que um instrumento de avaliação psicológica seja considerado válido, é imprescindível que o mesmo tenha uma boa precisão (Wright \& Stone, 2004). Durante o estudo de evidências de validade psicométricas de dado instrumento, é essencial a avaliação da fidedignidade do mesmo. Existem diferentes métodos para avaliação da fidedignidade. Dentre os principais, está o coeficiente de alfa de Cronbach, que é capaz de mensurar a consistência interna tanto da escala como um todo, como de suas subescalas (Hair \& cols., 2009).

Após a eliminação dos itens descritos acima, a consistência interna da URICA-24 itens foi analisada por meio do coeficiente de alfa de Cronbach. $\mathrm{Na}$ Tabela 2, podem ser observados os valores de alfa para cada fator, da versão reduzida, assim como da escala total.

Tabela 2. Alfa de Cronbach URICA versão 24 Itens ( $\mathrm{n=175)}$

\begin{tabular}{lc}
\hline Fatores & Alfa de Cronbach* \\
\hline Fator 1 - Ação & 0,940 \\
Fator 2 - Contemplação & 0,821 \\
Fator 3 - Pré-Contemplação & 0,822 \\
Fator 4 - Manutenção & 0,813 \\
\hline Total & 0,842 \\
\hline
\end{tabular}

*Coeficiente de alfa de Cronbach, com nível de significância de 5\%

\section{Discussão}

Por meio da análise fatorial exploratória, observou-se que a URICA 24 itens ficou agrupada em 4 fatores, segundo a estrutura original da escala. Os 4 itens explicaram, conjuntamente, $57,55 \%$ da variância total observada, o que é fortemente corroborado pelos resultados do estudo original, onde a estrutura de 4 fatores encontrada explicou 58\% da variância observada (McConnaughy \& cols., 1983).

Neste estudo, o Fator 1 (Ação) foi responsável por $30,12 \%$ da variância total e todos os itens retidos apresentaram cargas altas (maiores que 0,80), além de terem carregado somente neste fator. 0 Fator 2 (Contemplação) foi responsável por $13,27 \%$ da variância total e, dos itens retidos, 3 obtiveram cargas altas (maiores que 0,70) no fator. Os outros 3 apresentaram cargas maiores que 0,50, porém, também carregaram em outros fatores. 0 item 4 apresentou carga de 0,67 no Fator 2 (Contemplação) e, ao mesmo tempo, de 0,33 no Fator 1 (Ação) e -0,30 no Fator 3 (Pré-Contemplação). 0 item 19 apresentou carga de 0,60 no Fator 2 e de 0,48 no Fator 4 (Manutenção). Finalmente, 0 item 21 apresentou carga de 0,51 no Fator 2 e de 0,31 no Fator 4. Em todos os casos, os itens carregaram mais fortemente no domínio que eles se destinam a avaliar. Teoricamente, uma vez que os estágios são correlacionados, as cargas simultâneas em outros fatores fizeram sentido. Segundo Pasquali (2010), quando se supõe que os fatores sejam correlacionados, é esperado que um mesmo item carregue em mais de um fator simultaneamente, com níveis de saturação diferentes em cada fator.

0 Fator 3 (Pré-Contemplação), respondeu por 9,69\% da variância total e, dos seis itens retidos, 4 obtiveram cargas altas (de 0,69 a 0,77) e somente carregaram nesse fator. 0 s itens 5 e 1 obtiveram cargas de 0,59 e 0,54 (respectivamente) no Fator 3 , ao mesmo tempo em que apresentaram cargas negativas no Fator 2 (Contemplação), de -0,46 e -0,39. Nos dois casos, os itens carregaram mais fortemente no fator que eles se propõem a avaliar.

Finalmente, o Fator 4 (Manutenção) respondeu a $4,41 \%$ da variância total, sendo que todos os itens retidos, apesar de carregarem mais fortemente nesse fator, também apresentaram cargas significativas no Fator 2 (Contemplação) e 1 (Ação). Essas correlações fazem sentido do ponto de vista teórico, pois a presença de ambivalência do estágio de manutenção é um fato, sendo um dos cuidados indicados para os terapeutas de pacientes em fase de manutenção, que se utilizam da abordagem da prevenção à recaída, que estes não subestimem a presença da ambivalência nesse estágio (Brandon, Vidrine \& Litvin, 2007; Hendershot, Witkiewitz, George \& Marlatt, 2011). Ou seja, não é porque a pessoa está mantendo a mudança do comportamento problema que ela não pode ficar em dúvidas se isso realmente é necessário. A correlação entre 0 estágio de ação e de manutenção também é esperada, uma vez que o estágio de Manutenção nada mais é do que manter as mudanças iniciadas na fase de ação (DiClemente, 2003).

$\mathrm{Na}$ maioria dos casos em que os itens de cada fator foram excluídos, se tratava de itens que não apresentaram cargas iguais ou maiores 0,30 nos fatores avaliados. Ao mesmo tempo, também se evidenciou que em alguns fatores, o item carregou em outro(s) domínio(s) que não o avaliado. Dificuldade semelhante foi encontrada por Dozois e colaboradores (2004), que em sua discussão acerca das evidências encontradas na 
análise fatorial da URICA, afirmam que itens que originalmente seriam de uma subescala carregaram em outras.

A fidedignidade encontrada na URICA 24 itens deste estudo, segundo o alfa de Cronbach, foi de $a=0,940, a=0,821, a=0,822$ e $a=0,813$, para Ação, Contemplação, Pré-Contemplação e Manutenção, respectivamente. Os 24 itens tiveram um Alpha de 0,842. D ois estudos de McConnaughy e colaboradores (1983 e 1989) encontraram boa consistência interna na URICA, a qual variou de 0,79 a 0,89 para as subescalas. $\mathrm{Na}$ validação norueguesa da URICA para exercícios físicos (Lerdal \& cols., 2009), a consistência interna encontrada nas diferentes subescalas variou de 0,72 a 0,92 (alfa de Cronbach). Dozois e colaboradores (2004), em seu estudo de validação da URICA para pessoas com problemas relacionados à ansiedade, encontraram uma consistência interna de $\alpha=0,79$, sendo que os alfas entre as diferentes subescalas foram de $0,77,0,88,0,84$ e 0,82 para $\mathrm{PC}, \mathrm{C}, \mathrm{A}$ e $\mathrm{M}$, respectivamente.

Hasler e colaboradores (2003) realizaram estudo de validação da URICA para transtornos alimentares em uma amostra alemã, onde a consistência interna encontrada foi de $\alpha=0,86$. Na validação brasileira da URICA para substâncias psicoativas ilícitas (Szpszynski \& O liveira, 2008), a consistência interna, na escala de 24 itens, foi de $\alpha=0,657$ e variou de 0,71 a 0,81 e na validação brasileira da URICA para dependentes de álcool ambulatoriais encontraram-se alfas de Conbrach variando de 0,63 a 0,80 (Figlie \& cols., 2005). Assim, em comparação com os demais estudos, observa-se que a fidedignidade deste estudo, tanto nas 4 subescalas, como na URICA total, está dentro ou acima do que era esperado. Segundo Urbina (2007), um alfa de Cronbach de 0,70 indica que os itens de uma escala avaliam 0 mesmo construto de forma consistente, sendo que um coeficiente igual ou maior a 0,80 é considerado bom.

\section{Considerações finais}

Esta pesquisa identificou boas propriedades psicométricas para a URICA 24 itens para mudança de hábitos alimentares, com uma solução de 4 fatores, 0 que possibilita a utilização da escala na prática clínica e de pesquisa na realidade brasileira. A manutenção de hábitos alimentares saudáveis é um desafio, uma vez que as recaídas são frequentes e não é possível a abstinência total de alimentos. O presente estudo, através da validação da URICA para mudança de hábitos alimentares, possibilitará uma avaliação adequada dos estágios de motivação em que os indivíduos se encontram, com relação à mudança de seus hábitos alimentares. Percebe-se a necessidade de dar prosseguimento ao desenvolvimento e busca de evidências de validade de instrumentos de avaliação precisos, que avaliem comportamentos específicos, a fim de oportunizar 0 uso de estratégias clínicas mais eficazes.

\section{Referências}

Amodei, N., \& Lamb, R. J. (2004). Convergent and concurrent validity of the contemplation Ladder and URICA Scales. Dnug and Alchd Dependence, 73(3), 301-306.

ANEP - Associação Nacional de Empresas de Pesquisa. (2008). C Critéio de Classificação Econômica Brasil. Recuperado: 10 de outubro de 2010. Disponível: http:/ / www.anep.org.br

Belding, M. A., Iguchi, M. Y., \& Lamb, R. J. (1996). Stages of change in methadone maintenance: assessing the convergent validity of two measures. Psydhdogy of AddidiveBdhaviors, 10(3), 157-166.

Brandon, T. H., Vidrine, J. I., \& Litvin E. B. (2007). Relapse and relapse prevention. Annul Review of Clinical Psydhdogy, 3, 254-284.

Carbonari, J. P., \& DiClemente, C. C. (2000). Using theoretical model profiles to differentiate levels of alcohol abstinence success. Jaumal of Consultingand Clinical Psychdogy, 68(5), 810-817.

Carbonari, J. P., DiClemente, C. C., \& Zweben, A. (1994). A readinss to dange measure San Diego: Association for the Advancement of Behavior Therapy.

Carney, M. M., \& Kivlahan, D. R. (1995). Motivational subtipes among veterans seeking substance abuse treatment profiles based on stages of change. Psychdogy of AddidiveBdhaviars, 9(2), 135-142.

Carretero-Dios, H., \& Pérez, C. (2007). Standards for the development and the review of instrumental studies: considerations about test selection in psychological research. Intemational Jaumal of Clinical and HealthPsychdogy, 7(3), 863-882.

DiClemente, C. C. (2003). Addiction and dange how addictions deudop and addicted peeple reover. Nova Iorque: Guilford Press.

Dozois, D. J., Westra, H. A., Collins, K. A., Fung, T. S., \& Garry, J. K. (2004) Stages of change in anxiety: psychometric properties of the University of Rhode Island Change Assessment Scale (URICA). Behaviour R 729 . 
D unn, E. C., Neighbors, C., \& Larimer, M. E. (2003). Assessing readiness to change binge eating and compensatory behaviors. Eating Bdhaviors, 4(3), 305-314.

Figlie, N. B., Dunn, J., Bazan, J. L., \& Laranjeira, R. (2005). The stages of change scale among brazilian alcohol dependents. Addidive Disardas \& Ther Treatment, 4(4), 161-165.

Finucane, M. M., Stevens, G. A., Cowan, M. J., Danaei, G., Lin, J. K., Paciorek, C. J., Singh, G. M., Gutierrez, H. R., Lu, Y., Bahalim, A. N., Farzadfar, F., Riley, L. M., \& Ezzati, M. (2011). National, regional, and global trends in body-mass index since 1980: systematic analysis of health examination surveys and epidemiological studies with 960 country-years and 9.1 million participants. Lance, 377, 557-567.

Hair, J. F., Black, W. C., Babin, B. J., Anderson, R. E., \& Tatham, R. L. (2009). Análise miltivariada dos dados Porto Alegre: Bookman.

Hasler, G., Klaghofer, R., \& Buddeberg, C. (2003). The University of Rhode Island Change Assessment Scale (URICA) psychometric testing of a G erman version. Psychtherapie Psychosomatik Medizinishe Psychdoge, 53(10), 406-411.

Hendershot, C. S., Witkiewitz, K., George, W. H., \& Marlatt, G. A. (2011). Relapse prevention for addictive behaviors. Substance Abuse Treatment, Presention, and Pdigy, 6, 17-33.

Lerdal, A., Moe, B., Digre, E., Harding, T., Kristensen, F., Grov, E. K., Bakken, L. N., Ruud, I., \& Rossi, J. S. (2009). Stages of change continuous measure (URICA-E2): psychometrics of a Norwegian version. Jaumal of Adxanced Nursing 65(1), 193-202.

Levesque, D. A., Gelles, R. J., \& Velicer, W. F. (2000). D evelopment and validation of a stages of change measure for men in batterer treatment. Cogitive Therapyand Reserth, 24(2), 175-199.

McConnaughy, E. A., Prochaska, J. O., \& Velicer, W. F. (1983). Stages of change in psychotherapy: measurement and sample profiles. Psydhterapy: Thery, Reserch \& Pradice, 20(3), 368-375.

McConnaughy, E. A., DiClemente, C. C., Prochaska, J. O., \& Velicer, W. F. (1989). Stages of change in psychotherapy: a follow-up report. Psychotheapy, Theory, Rearch Practice Training 26(4), 494-503.

Montero, I., \& León, O. G. (2005). Sistema de clasificación del método en los informes de investigación en Psicología. Intemational Jaumal of Clinical and HealthPsychdogy, 5(1), 115-127.
Nunes, C. H. S. S., \& Primi, R. (2010). Aspectos técnicos e conceituais da ficha de avaliação dos testes psicológicos. Em Conselho Federal de Psicologia (O rg.), Avaliação psiclógica: diretrizes na regulamentação da profissão (pp. 101-128). Brasília: CFP.

Oliveira, M. S., Freire, S. F., \& Cazassa, M. J. (2007). Validação da versão brasileira da escala URICA para abuso ou dependência de álcool. Anais do XIX Congesso da ABEAD, Rio de Janeiro, Rio de Janeiro, Brasil.

Pantalon, M. V., Nich, C., Franckforter, T., \& Carroll, K. M. (2002). The URICA as a measure of motivation to change among treatment-seeking individuals with concurrent alcohol and cocaine problems. Psychdogy of Addidive Bdnaviors, 16(4), 299-307.

Pasquali, L. (2010). Testes referentes a construto: teoria e modelo de construção. Em L. Pasquali (Org.), Instrumetação psicoógica: fundamentos e práticas (pp. 165-198). Porto alegre: Artmed.

Rodrigues, V. S., Oliveira, M. S., Ludwig, M. W. L., Cazassa, M. J., \& Fernandes, R. (2007). Medida de fidedignidade da University of Rhode Island Change Assessment (Urica) para dependentes de tabaco. AnaisdoXIX CongressodaABEAD, Rio de Janeiro, Rio de Janeiro, Brasil.

Rossi, S. R., Greene, G. W., Rossi, J. S., Plummer, B. A., Benisovich, S. V., Keller, S., Velicer, W. F., Redding, C. A., Prochaska, J. O., Pallonen, U. E., \& Meier, K. S. (2001). Validation of decisional balance and situational temptations measures for dietary fat reduction in a large school-based population of adolescents. EatingBenaviass 2(1), 118.

Siegal, H. A., Li, L., Rapp, R. C., \& Saha, P. (2001). Measuring readiness for change among crack cocaine users: a descriptive analysis. Substance Use andMisuse, 36(7), 687-700.

Souza, C. C., Silva, J. G., \& Oliveira, M. S. (no prelo). Evidências psicométricas da University Rohde Island Change Assessment (URICA) para o jogo patológico. Revista dePsiquiatria Clínica

Steinhausen, H. C. (2009). Outcome of eating disorders. Child Addescent Psychiatric Clinics Noth America, 18(1), 225-242.

Szupszynski, K., \& Oliveira, M. (2008). Adaptação brasileira da University of Rhode Island Change Assessment (URICA) para usuários de substâncias ilícitas. PsicoUSF, 13(1), 31-39. 
Urbina, S. (2007). Fundamentos da Testagem Psiclógica Porto Alegre: Artmed.

Velasquez, M. M., Maurer, G. G., Crouch, C., \& DiClemente, C. C. (2001). Group treatment for substanceabuse a stagss of-dhangetherapy manul. Nova Iorque: Guilford Press.

Velicer, W. F., DiClemente, C. C., Prochaska, J. O ., \& Brandenburg, N. (1985). D ecisional balance measure for assessing and predicting smoking status. Joumal of Pessonality and Social Psychdogy, 48(5), 1279-1289.

Willoughby, F. W., \& Edens, J. F. (1996). Construct validity and predictive utility of the stages of change scale for alcoholics. Jaumal of Substance Abuse, 8(3), 275-291.
WHO - World Health Organization (2000). Obesity: preventing and managing the global epidemic. Report of a World Health Organization Consultation. Wold Health Organization Technical Repart Series, 894(i-xii), 1-253.

WHO - World Health Organization (2006). BMI Classification Recuperado: 10 de outubro de 2010. Disponível:

http:/ / apps.who.int/ bmi/ index.jsp?introPage=int ro 3.html.

Wright, B. D., \& Stone, M. H. (2004). Makingmasure Chicago: The Phaneron Press.

Reedidbem25/ 10/ 2011

Refomuladbem22/08/2012

Aprovadbem08/ 09/ 2012

Sobre as autoras:

Simone Armentano Bittencourt é psicóloga graduada pela Pontifícia Universidade Católica do Rio Grande do Sul (PUCRS), mestre e doutora em Psicologia pela PUCRS e professora do Instituto Cyro Martins.

Paola Lucena dos Santos é psicóloga graduada pela PUCRS, doutoranda em Psicologia na Universidade de Coimbra/ Portugal e bolsista de D outorado Pleno no Exterior pela CAPES (Coordenação de Aperfeiçoamento de Pessoal de Nível Superior).

Margareth da Silva Oliveira é psicóloga graduada pela PUCRS, mestre em Psicologia (PUCRS), doutora em Ciências da Saúde (Universidade Federal de São Paulo), bolsista de Produtividade em Pesquisa (Conselho Nacional de Desenvolvimento Científico e Tecnológico - CNPq) e professora Adjunta da Faculdade de Psicologia da PUCRS.

Contato com as autoras:

Avenida Ipiranga, 6.681, Prédio 11, sala 927 - Bairro Partenon - CEP: 90619-900. Porto Alegre-RS.

E-mail: marga@ pucrs.br 